doctor, patient and parent, they are probably avoidable in the majority of cases. However, prevention is better than cure and the foresighted use of witnesses, chaperones, and second opinions may forestall them. Clear, decipherable communication and contemporaneous notes are imperative to allow doctors and their advisors to conduct a robust defence in any subsequent complaint or allegation.

1 Silber TJ. False allegations of sexual touching by physicians in the practice of pediatrics. Pediatrics 1994; 94: 742-5.

2 Kaplan J. False allegations of sexual touching. Pediatrics 1995; 95: 797.

3 Ricci LR. False allegations of sexual touching. Pediatrics 1995; 95: 797 .

4 Silber TJ. False allegations of sexual touching. Pediatrics 1995; 95: 797-8.
5 Gillick v West Norfolk and Wisbech Area Health Authority [1986] AC 112; [1985] 3 All ER 402; [1985] 3 WLR 830 6 Section 8 of the Family Law Reform Act 1969, page 5 re C (mental patient: medical treatment) [1993] BMLR 15, 77, fam $D$.

7 Sidaway. The Governors of Royal Bethnal Hospital [1985] 1 All ER 643 .

8 Neinstein LS, Shapiro J, Rabinovitz S, Baer K, Church J, Sasao T. Comfort of male adolescents during general and genital examination. $\mathcal{F}$ Pediatr 1989; 115: 494-7.

$9 \operatorname{Re} \mathrm{R}$ (a minor) (wardship: medical treatment), re [1991] 4 All ER 177.

10 Devereux JA, Jones DPH, Dickenson DL. Can children withhold consent to treatment? $B M \mathcal{F}$ 1993; 306: 1459-61. withhold consent to treatment? BMf 1993; 306: 1459-61.
Elton A, Honia P, Bentovim A, Simons J. Withholding consent to lifesaving treatment: three cases. BMF 1995; 310: 373-7.

12 Shield JPH, Baum JD. Children's consent to treatment. BMF 1994; 308: 1182-3.

13 Gaylin $\mathrm{W}$. The competence of children: no longer all or none. Hastings-on-Hudson, New York: Hastings Center Report, 1982; April: 33-8.

14 American Academy of Pediatric Committee on Bioethics. Informed consent, parental permission and assent in pediatric practice. Pediatrics 1995; 95: 314-7.

15 General Medical Council. Good medical practice. Duties of a doctor. London: General Medical Council, 1995: paras 18,19 .

\title{
When is HIV an issue after child sexual abuse?
}

\author{
Jacqueline Y Q Mok
}

Child sexual abuse is an increasingly recognised problem, the complications of which include a small, but real risk of acquiring infection with HIV. The incidence of HIV infection acquired by children through sexual abuse is unknown. Sexual abuse does not appear as a separate transmission category among the cases of HIV infection reported to the British Paediatric Association Surveillance Unit. However, there is a rising incidence of young adults reported with HIV infection in the UK, some of whom could have acquired the infection as a result of sexual abuse. Children and young people have an awareness of HIV and AIDS and are taught about harm minimisation and healthy living to reduce the risk of HIV infection. Health promotion and personal safety programmes in schools have, in turn, led to disclosures of abuse in children. Sexual abuse and HIV evoke strong emotive reactions, but there are also difficult and complex issues that need to be addressed. The greatest demand for HIV testing comes from professionals working with children and adolescents who have been sexually abused, including vulnerable young people who may be survivors of abuse. As yet, most guidelines on child protection do not discuss whether HIV testing should be performed, although some professionals working with children who have been sexually abused have made some recommendations on HIV testing. ${ }^{2}$ Recent government guidance on children and HIV also raises the possibility of HIV infection where children have been sexually abused. ${ }^{3}$

\section{Are the concerns valid?}

Sexual abuse is a documented mode of transmission of HIV, although reports are anecdotal. ${ }^{45}$ It is conceivable that traumatic abuse involving penetration would increase the risk of HIV transmission by mucosal breach, and the immature anogenital tract might be more vulnerable to infection. In an attempt to determine the incidence of HIV infection acquired through sexual abuse, a telephone survey was conducted of 63 practitioners who see sexually abused children in five regions in the United States with the highest prevalence of HIV infection. No formal protocol was in place for HIV antibody testing of abused children. In approximately $1000 \mathrm{HIV}$ antibody tests performed by the practitioners, HIV infection was discovered in two children in whom no other cause for infection was identified. ${ }^{6}$ In a further survey of 2147 health and social service professionals involved with child abuse assessment, the authors found that 5622 HIV antibody tests were conducted during 113198 sexual abuse assessments. Twenty eight children were found to be infected with HIV and had no alternative transmission route other than sexual abuse, a crude rate of 0.25 per 1000 assessments.

Although most children who have been abused cope well with no long term effects, it is acknowledged that for many survivors, the experience can result in activities which may predispose the young person to HIV infection. ${ }^{89}$ Characteristics of the child sexual abuse survivor have included sexual compulsivity, revictimisation, and low self esteem with chronic depression, ${ }^{10}$ which may lead to alcohol and drug abuse, exposing the adolescent to HIV infection. On the other hand, recognised high risk behaviours for HIV infection are found among many homeless teenage 
runaways as well as emotionally and behaviourally disturbed young people, who are likely to be survivors of sexual abuse. ${ }^{11}$

A study that explored the health and social needs of children who had been sexually abused found that concerns regarding HIV often existed, although they were not always voiced. ${ }^{12}$ Professionals who work with children who have been abused should be prepared to listen to, rather than deny, the concerns.

When should HIV counselling be offered? At present HIV counselling is not a routine procedure in the assessment of children who have suffered sexual abuse. A recent article in this journal highlighted the therapeutic importance of the paediatric assessment, but failed to discuss whether HIV counselling should be offered..$^{13}$ In an effort to develop guidelines for HIV antibody testing in children who have been the subject of sexual abuse, Gellert found that among paediatricians surveyed, there was an $85 \%$ consensus for six criteria for HIV antibody testing, namely where the assailant was known to be HIV infected, had clinical findings suggestive of HIV infection, or was known or suspected to engage in high risk behaviour, if the child had clinical findings compatible with HIV infection, where there was a history of rectal penetration, or where the young person had a history of high risk behaviour. Some also agreed that abuse by multiple assailants should prompt HIV counselling, as should a direct request from the parent, carer, or young person. There was less agreement as to whether testing should be performed where there was a coexisting sexually transmitted disease, where there was an unknown single assailant, or whether vaginal penetration should automatically raise the possibility of HIV infection. ${ }^{6}$

Does knowledge about the perpetrator or type and frequency of abuse help in the assessment? In 28 children found to be infected with HIV, 16 of 24 perpetrators were known to be infected with HIV during the time of abuse. The forms of abuse suffered by the children included penile-vaginal penetration in $65 \%$, and penile-rectal penetration in $50 \%$, occurring over one week to seven years' duration. Although 13 of 19 children had more than six episodes of abuse, three of the victims reported only a single episode. Nine children had another sexually transmitted disease.

\section{Difficulties establishing HIV transmission}

If the abuse was acute an early specimen, taken before seroconversion, would be HIV antibody negative. As most cases of sexual abuse occur over a long period, this difficulty may not arise. Rather than subject the child to testing, some professionals have proposed testing of the alleged perpetrator. However this is fraught with legal and ethical issues regarding infringement of civil liberties, which usually leaves the paediatrician having to test the child. Where possible, a request should be made to the crime laboratory for access to any stored samples of serum from the alleged perpetrator, for future use. Additional problems are posed when sexually active youngsters may already be infected with HIV, making interpretation of the result difficult.

\section{Should HIV testing be part of the} assessment?

For adult victims of sexual assault, HIV counselling is an integral part of the service, and a similar level of service should be provided for children. HIV testing meets many criteria for a screening test in that a serious condition is detected, an accurate predictive test is available, and interventions are now practicable. The cost/benefit ratio is probably acceptable, given the costs incurred in caring for a child with HIV infection. Arguments against testing, however, are that there is still no definitive cure, and families may not welcome unnecessary medical attention. The social stigma and trauma experienced by many families living with HIV unfortunately still exist. Also, professionals who do not possess counselling skills or knowledge about HIV transmission or testing could struggle with their own anxieties, and add to the burden of the child and family.

\section{Recommendations}

LISTENING TO THE CONCERNS

Where the young people, parents, or carers have questions regarding HIV after sexual abuse, these should be dealt with sensitively, appropriately, and without delay. Therefore all staff working with children who have suffered sexual abuse should develop their own knowledge of HIV, including the routes of transmission, ways of prevention, HIV support services, and advances in treatment. Only then can the concerns be dealt with in a balanced way. On the other hand clinicians working with HIV infected young people must be aware of the various forms of child abuse, including the psychosocial characteristics of survivors of abuse. The apparent link between unresolved child abuse and increased vulnerability to HIV infection highlights the need for closer cooperation between professionals working in separate specialties.

\section{RISK ASSESSMENT}

The need for HIV testing will vary according to the prevalence of HIV infection in the area, as well as the context in which abuse occurs. A careful history may reveal potential risk factors in the perpetrator which might make HIV testing more pertinent. These would include:

- HIV infection

- Injecting drug misuser

- Bisexual practices

- Multiple sexual partners

For the child or young person, HIV testing should be considered where the following were identified:

- Multiple assailants

- Presence of a sexually transmitted disease

- History of high risk behaviour

- History of rectal or vaginal penetration

- Parent or young person requests testing 
FUTURE DIRECTIONS

With the heterosexual spread of HIV, more victims of sexual abuse will be put at risk of acquiring the infection, especially in areas of high prevalence. The British Paediatric Association Surveillance Unit should consider sexual abuse as a separate transmission category for HIV infection, in an attempt to elucidate the extent of the problem. There must also be professional recognition and acceptance of the need to obtain support and training for themselves.

1 Public Health Laboratory Service AIDS Centre and the Scottish Centre for Infection and Environmental the Scottish Centre for Infection and Environmental Noalth. 28.

2 Hobbs CJ, Hanks HIG, Wynne JM, eds. Child abuse and neglect. A clinician's handbook. Edinburgh: Churchill Livingstone, 1993: 207-12.

3 The Scottish Office. Children and HIV. Guidance for local authorities and voluntary organisations. Edinburgh: The Scottish Office, 1993.
4 Leiderman A, Teets Grimm K. A child with HIV infection (letter). $\mathscr{F} A M A$ 1986; 256: 3094.

5 Rubinstein A. Pediatric AIDS. Curr Probl Pediatr 1986; 16: 362.

6 Gellert GA, Durfee MJ, Berkowitz CD. Developing guidelines for HIV antibody testing among victims of pediatric sexual abuse. Child Abuse Negl 1990; 14: 9-17.

7 Gellert GA, Durfee MT, Berkowitz CD, Higgins KV, Tubiolo VC. Situational and sociodemographic characteristics of children infected with HIV from pediatric sexual abuse. Pediatrics 1993; 91: 39-44.

8 Allers CT, Benjack KJ, White J, Rousey JT. HIV vulnerability and the adult survivor of childhood sexual abuse. Child ity and the adult survivor of
Abuse Negl 1993; 17: 291-8.

9 Allers CT, Benjack KJ. Connections between childhood abuse and HIV infection. Fournal of Counselling and Development 1991; 70: 309-13.

10 Browne A, Finkelhor D. Impact of child sexual abuse: a review of the research. Psychol Bull 1986; 99: 66-77.

11 Carmen E, Reiker PR. A psychosocial model of the victimto-patient process. Psychiatr Clin North Am 1989; 12: 43143.

12 Thomas A. Health and social needs assessment. Child sexual abuse and HIV-AIDS. Report to Barnardo's Yorkshire and Humberside Division and Wakefield Health Care, March 1994.

13 Lazaro CDS. Making paediatric assessment in suspected sexual abuse a therapeutic experience. Arch Dis Child 1995; 73: 174-6. 